\title{
MODELO DE NEGÓCIO DE SISTEMAS PRODUTO-SERVIÇO: UMA ANÁLISE DA LITERATURA
}

Suzana R. Moro (suzana.moro19@gmail.com) - Programa de Pós-graduação em Engenharia de Produção, Universidade Federal de Santa Catarina (UFSC).

Paulo A. Cauchick-Miguel (paulo.cauchick@ufsc.br) - Departamento de Engenharia de Produção e Sistemas, Universidade Federal de Santa Catarina (UFSC).

\section{RESUMO}

O sistema produto-serviço (product-service system - PSS) pode ser considerado um modelo de negócio com capacidade para gerar rentabilidade e competitividade para as organizações, além de contribuir com a sustentabilidade. No entanto, é necessário que as empresas desenvolvam novas competências para o desenvolvimento e implementação de modelos de negócio com foco em PSS. Além disso, é necessário que o modelo de negócio seja capaz de gerar valor, trazendo benefícios para os clientes. Nesse contexto, este trabalho compreende uma análise da literatura para identificação de características desse tipo de modelo de negócio, componentes e mecanismos para adição de valor ao PSS. Foram selecionadas 65 publicações relacionadas com modelo de negócio e PSS. Por meio da análise das publicações foram identificados 14 componentes para serem considerados ao estruturar um modelo de negócio de PSS, que estão associados a mecanismos de proposição, criação, entrega, e captura de valor. Desenvolver um modelo de negócio de PSS requer a formação de uma rede de valor com propósitos alinhados, considerando os interesses e responsabilidades dos envolvidos para a geração de valor mútuo.

Palavras chave: sistema produto-serviço, servitização, modelo de negócio sustentável, revisão de literatura 


\section{INTRODUÇÃ̃}

Em resposta a diversas transformações que vêm ocorrendo na última década, como a globalização, o aumento da concorrência com base em baixo custo, cadeias e redes de empresas guiadas pela demanda (VAN HALEN et al., 2005), as empresas estão cada vez mais percebendo a necessidade de readaptar suas estratégias com foco na geração de valor para os clientes (FRANÇA et al., 2017). A indústria automotiva, por exemplo, tradicionalmente baseada na venda do meio de transporte, vem investindo em pesquisas sobre novas formas de ofertar mobilidade focando no fornecimento de valor ao consumidor na fase de uso do produto (MAHUT et al., 2017). No entanto, a transição para ofertas com foco no desempenho dos produtos exige o desenvolvimento de modelos de negócio inovadores (EVANS et al., 2017). A abordagem de sistemas produto-serviço (product-service system - PSS) é focada em novos modelos de negócio que atendem as necessidades funcionais do cliente de maneira inovadora, adicionando serviços aos produtos ofertados (SCHEEPENS et al., 2016). Esta abordagem foi proposta como uma oportunidade para promover a sustentabilidade e o desenvolvimento estratégico do modelo de negócio (TUKKER, 2004; FRANÇA et al., 2017), sendo uma solução para entregar valor aos clientes (REIM et al., 2015). Sendo assim, para a oferta "produtoserviço' geralmente é desenvolvido um modelo de negócio inovador, apoiado no conceito de sustentabilidade (BOCKEN et al., 2014). Desenvolver um PSS ou transformar um modelo de negócio existente em PSS tem sido descrito como inovação de modelo de negócio para a sustentabilidade (EVANS et al., 2017). Embora o PSS como um tópico de pesquisa tenha surgido durante os anos 90, continua atraindo interesse, com a quantidade de publicações em constante crescimento, provavelmente devido à convergência de causas específicas ao longo dos anos, como a disseminação da tecnologia de comunicação e informações e seu amplo emprego nas organizações industriais (ANNARELLI et al., 2016).

No entanto, muitas empresas vêm enfrentado dificuldades para desenvolver seu modelo de negócio como um PSS (REIM et al., 2015) e também combinar a sustentabilidade com a inovação (FRANÇA et al., 2017). Nesse sentido, esse trabalho objetiva analisar a literatura sobre PSS sob a ótica do conceito de modelo de negócio, visando identificar os componentes necessários para que as empresas desenvolvam seu modelo de negócio de PSS com foco na geração de valor. 


\section{CONCEITOS RELACIONADOS A MODELO DE NEGÓCIO}

O conceito de modelo de negócio difundiu-se exponencialmente após um trabalho co-criado por 470 praticantes de 45 países (OSTERWALDER; PIGNEUR, 2010), elaborado principalmente com base em Osterwalder (2004), que aborda a ontologia do modelo de negócio. O autor define o termo 'business model' como (OSTERWALDER, 2004, p. 14): "uma representação de como uma empresa compra e vende bens e serviços e ganha dinheiro". O modelo de negócio conecta o potencial técnico com a realização do valor econômico (CHESBROUGH; ROSENBLOOM, 2002). No que diz respeito a dimensão operacional, a essência do conceito ‘modelo de negócio' já era apresentada por Peter Drucker na década de 50, que é um clássico a para o estudo da eficiência dos negócios (FJELDSTAD; SNOW, 2018). São importantes contribuições conceituais as publicações de Chesbrough e Rosenbloom (2002) e Teece (2010), além de Osterwalder et al. (2005). Mesmo assim, há ainda uma falta de clareza, consenso conceitual e consistência no uso dos termos modelo de negócio, inovação do modelo de negócio e modelos de negócio sustentáveis (OSTERWALDER et al., 2005; BOONS; LÜDEKE-FREUND, 2013; EVANS et al., 2017).

Embora seja possível conceber modelos de negócio genéricos, cada empresa tem seu modelo de negócio único, que descreve como a empresa cria e captura valor (KINDSTRÖM; KOWALKOWSKI, 2014). De acordo com essa perspectiva, cada empresa deve estruturar seu modelo de negócio individualmente para adequar-se à estratégia e operações da organização (TUKKER, 2015).

O processo de mudar um modelo de negócio, por sua vez, constitui um processo de inovação no modelo de negócio (KINDSTRÖM; KOWALKOWSKI, 2014; FJELDSTAD; SNOW, 2018). Diferentemente da inovação de produtos ou serviços, a inovação do modelo de negócio não necessariamente descobre um novo produto ou serviço, mas usa novas maneiras de criar e entregar o produto ou serviço existente e novas maneiras de capturar o valor dele (YANG et $a l .$, 2017). O desenvolvimento de um modelo de negócio consiste na estruturação de diversos componentes (também denominados elementos, funções ou atributos do modelo de negócio) que são descritos a seguir. 


\subsection{Componentes dos modelos de negócio de PSS}

Após a análise e contraste entre os componentes do modelo de negócio sob a ótica de diversos autores, Osterwalder (2004) descreve nove componentes que cobrem quatro principais áreas de um negócio: (i) clientes, (ii) oferta, (iii) infraestrutura, e (iv) viabilidade financeira. Osterwalder e Pigneur (2010) apresentam os componentes estruturados na forma de um quadro denominado 'Business Model Canvas', denominado no presente trabalho de modelo Canvas. Os componentes são ilustrados na Figura 1 conforme a descrição dos autores previamente citados.

\begin{tabular}{|c|c|c|c|c|}
\hline $\begin{array}{l}\text { PARCERIAS } \\
\text { PRINCIPAIS }\end{array}$ & $\begin{array}{c}\text { ATIVIDADES- } \\
\text { CHAVE } \\
\text { Mais importantes ações } \\
\text { que a organização precisa } \\
\text { tomar para que o modelo } \\
\text { de negócios funcione }\end{array}$ & $\begin{array}{l}\text { PROPOSTA DE } \\
\text { VALOR }\end{array}$ & $\begin{array}{l}\text { RELACIONAMENTO } \\
\text { COM CLIENTES } \\
\text { Tipos de relacionamentos } \\
\text { que são estabelecidos e } \\
\text { mantidos com cada } \\
\text { segmento de clientes }\end{array}$ & \multirow{2}{*}{$\begin{array}{c}\text { SEGMENTOS } \\
\text { DE CLIENTES } \\
\text { Os diferentes } \\
\text { grupos de pessoas } \\
\text { ou organizações } \\
\text { que a empresa } \\
\text { pretende atingir }\end{array}$} \\
\hline $\begin{array}{l}\text { Rede de parceiros } \\
\text { e fornecedores } \\
\text { que fazem o } \\
\text { modelo de } \\
\text { negócios funcionar }\end{array}$ & $\begin{array}{l}\text { RECURSOS } \\
\text { PRINCIPAIS } \\
\text { Recursos mais importantes } \\
\text { para o funcionamento do } \\
\text { modelo de negócios }\end{array}$ & $\begin{array}{l}\text { Pacotes de produtos } \\
\text { e serviços que } \\
\text { buscam resolver os } \\
\text { problemas do cliente } \\
\text { e satisfazer suas } \\
\text { necessidades }\end{array}$ & $\begin{array}{c}\text { CANAIS } \\
\text { Canais para } \\
\text { comunicação, } \\
\text { distribuição e vendas }\end{array}$ & \\
\hline $\begin{array}{r}\text { ESTRUTL } \\
\text { CUST }\end{array}$ & $\begin{array}{r}\text { Custos env } \\
\text { na opera }\end{array}$ & $\begin{array}{l}\text { vidos } \\
\text { ão }\end{array}$ & $\begin{array}{c}\text { FONTES DE } \\
\text { RECEITA }\end{array}$ & $\begin{array}{l}\text { heiro que a } \\
\text { são gera com o } \\
\text { de negócio }\end{array}$ \\
\hline
\end{tabular}

FIGURA 1 - Componentes dos modelos de negócio. Fonte: adaptado de Osterwalder e Pigneur (2010)

Nota: foi acrescentado ao modelo Canvas a numeração sequencial e uma breve descrição de cada um dos componentes com base nos autores.

Os componentes do modelo de negócio mostrados na Figura 1 são interdependentes. Assim, uma mudança em um componente depende das mudanças correspondentes e/ou do realinhamento de outros componentes para que a inovação seja bem-sucedida (KINDSTRÖM; KOWALKOWSKI, 2014).

\subsection{Modelo de negócio sustentável}

Na maioria dos modelos de negócio, os princípios básicos estão relacionados ao conceito de valor definido por Evans et al. (2017) e Yang et al. (2017). De acordo com a perspectiva da 
sustentabilidade, os modelos de negócio normalmente consistem em uma proposição de valor, criação e entrega de valor e mecanismos de captura de valor (BOCKEN et al., 2014; SHORT et al., 2014).

Ao considerar as inovações do modelo de negócio para a sustentabilidade, existe uma maior complexidade associada à avaliação do impacto das inovações na sustentabilidade e como entender seus efeitos em toda a rede de negócios (EVANS et al., 2017). A ideia central de um modelo de negócio sustentável é uma proposta de valor sustentável, que permita a criação simultânea de valor para múltiplos stakeholders, incluindo clientes, acionistas, fornecedores e parceiros, bem como o meio ambiente e a sociedade (BOCKEN et al., 2014). Neste caso, exigese um pensamento mais integrado e a reconfiguração de vários aspectos do negócio, como capacidades, relações com os stakeholders, gestão do conhecimento, liderança e cultura, portanto envolvendo todo o modelo de negócio (EVANS et al., 2017).

Após estabelecer as bases do presente trabalho, a seção seguinte descreve os procedimentos metodológicos de pesquisa adotados no presente trabalho.

\section{PROCEDIMENTOS METODOLÓGICOS DE PESQUISA}

Para a execução desta revisão de literatura foram seguidos os procedimentos propostos por Booth et al. (2012). O Quadro 1 sintetiza os procedimentos adotados para a busca e seleção das publicações. Após a seleção das publicações, foi realizada uma análise da lista de referência utilizado o procedimento retrospectivo, que consiste em buscar estudos citados pelas publicações analisadas (GOUGH et al., 2012), uma vez que grande parte dos estudos identificados utilizam o termo modelo de negócio, mas não fazem a descrição dos componentes do mesmo.

Com base na análise das publicações selecionadas que apresentavam os componentes dos modelos de negócio (10 publicações) - foi feita uma comparação entre os componentes apresentados pelas mesmas (Quadros 2 e 3) e destacados os componentes que devem ser considerados ao estruturar um modelo de negócio de PSS. Por fim, foram descritos os mecanismos para adição de valor aos modelos de negócio de PSS por meio de: proposição, criação, entrega, e captura de valor (referente a 43 publicações analisadas). 
QUADRO 1 - Procedimentos adotados para a busca e seleção das publicações.

\begin{tabular}{|c|c|c|}
\hline \multicolumn{2}{|r|}{ Procedimentos } & Descrição \\
\hline \multirow{6}{*}{ 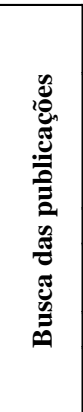 } & Propósito & $\begin{array}{l}\text { Conceituação dos modelos de negócio de PSS, descrição dos componentes dos modelos } \\
\text { de negócio de PSS e mecanismos para adição de valor do PSS }\end{array}$ \\
\hline & Palavras-chave & $\begin{array}{l}\text { Pesquisadas no título, palavras-chave e resumo das publicações= } \\
\text { Eixo } 1 \text { - PSS: "product-service system*" OR "product-extension service*” OR } \\
\text { "integrated product-service*" OR "product-service offer*” } \\
\text { Eixo 2 - Modelo de negócio: ("business model }{ }^{*} \text { ") }\end{array}$ \\
\hline & Bases de dados & Scopus, ISI Web of Science e Compendex (Engineering Village) \\
\hline & Período da busca & $\begin{array}{l}\text { Buscas realizadas entre abril e julho de } 2018 \text { - não foi utilizado filtro de data para as } \\
\text { publicações }\end{array}$ \\
\hline & Tipos de publicações selecionadas & Artigos publicados em periódicos revisados por pares em língua inglesa \\
\hline & $\begin{array}{l}\text { Quantidade de publicações } \\
\text { obtidas na busca }\end{array}$ & $\begin{array}{l}371 \text { publicações: Scopus - 122; ISI Web of Science - 113; e Compendex (Engineering } \\
\text { Village) - } 136\end{array}$ \\
\hline \multirow{5}{*}{ 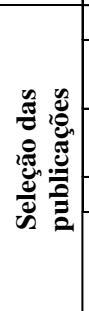 } & Total de não duplicadas & 220 publicações \\
\hline & Critérios para seleção & $\begin{array}{l}\text { Seleção das publicações conceituam e descrevem os modelos de negócios e seus } \\
\text { componentes }\end{array}$ \\
\hline & $\begin{array}{l}\text { Leitura do título, palavras-chave } \\
\text { e resumo }\end{array}$ & 74 publicações resultantes \\
\hline & Leitura na íntegra & 36 publicações resultantes \\
\hline & Procedimento retrospectivo & $\begin{array}{l}\text { Análise das referências das publicações selecionadas; inclusão de } 29 \text { publicações (livros } \\
\text { e publicações técnicas sobre modelo de negócio citadas pelos artigos selecionados } \\
\text { também foram consideradas) }\end{array}$ \\
\hline \multicolumn{2}{|c|}{ Total de publicações analisadas } & $\begin{array}{l}65 \text { (13 sobre modelo de negócio, } 9 \text { sobre modelo de negócio sustentável, e } 43 \text { sobre } \\
\text { modelo de negócio de PSS) }\end{array}$ \\
\hline
\end{tabular}

\section{RESULTADOS E DISCUSSÃO}

O assunto modelo de negócio de PSS ainda é recente na literatura. Das 36 publicações selecionadas sobre o assunto, mais da metade (58\%) são de 2016 em diante. Um PSS envolve uma mudança no mix produto-serviço, movendo-se de um sistema de produção 'baseado em recursos' para um sistema 'baseado em conhecimento' no qual as atividades comerciais visam atender as necessidades dos clientes (VAN HALEN et al., 2005). Portanto, é necessário que os modelos de negócio de PSS desenvolvidos considerem a adição de valor extra para as soluções, que dizem respeito principalmente à satisfação das necessidades dos clientes e custo (SCHEEPENS et al., 2016).

A adoção da abordagem de modelo de negócio fornece um entendimento mais abrangente para as empresas com o objetivo de alavancar, coordenar e alinhar com sucesso as transformações necessárias para a operacionalização (ADRODEGARI et al., 2017). Isso faz com que o modelo de negócio ofereça a oportunidade para fechar a lacuna entre o conhecimento sobre os efeitos das variáveis individuais no desempenho da empresa e a necessidade de conhecimento sobre como os relacionamentos entre essas variáveis afetam o desempenho (FJELDSTAD; SNOW, 2018). 


\subsection{Análise dos componentes dos modelos de negócio de PSS}

O Quadro 2 destaca as publicações selecionadas que descrevem os componentes dos modelos de negócio, bem como as contribuições e suas características.

QUADRO 2 - Contribuições dos modelos de negócio apresentados pela literatura.

\begin{tabular}{|c|c|c|c|c|c|}
\hline Publicação & $\begin{array}{c}\text { Foco } \\
\text { principal }\end{array}$ & Descrição & $\begin{array}{c}\text { Profundidade } \\
\text { de conteúdo }\end{array}$ & $\begin{array}{l}\text { \# tipos de } \\
\text { modelo de } \\
\text { negócio } \\
\text { descritos }\end{array}$ & $\begin{array}{l}\text { \# de } \\
\text { componentes } \\
\text { (Figura 1) }\end{array}$ \\
\hline $\begin{array}{l}\text { Osterwalder e } \\
\text { Pigneur } \\
(2010) \\
\end{array}$ & $\begin{array}{c}\text { Inovação em } \\
\text { modelos de } \\
\text { negócio }\end{array}$ & $\begin{array}{l}\text { Explica os padrões mais comuns do de modelos } \\
\text { de negócios }\end{array}$ & Detalhado & 5 & 9 \\
\hline $\begin{array}{l}\text { Meier et al. } \\
(2010) \text {; Meier } \\
\text { et al. }(2011)\end{array}$ & IPSS & $\begin{array}{l}\text { Descreve a organização modular e a rede de } \\
\text { atores envolvidos }\end{array}$ & Geral & 3 & 4 \\
\hline $\begin{array}{l}\text { Barquet et al. } \\
\quad(2013)\end{array}$ & IPSS & $\begin{array}{l}\text { Orienta a empresa na análise do contexto de } \\
\text { seus negócios, na escolha do tipo de PSS } \\
\text { adequado e na definição das características do } \\
\text { PSS }\end{array}$ & Detalhado & 4 & 9 \\
\hline $\begin{array}{c}\text { Boons e } \\
\text { Lüdeke- } \\
\text { Freund (2013) } \\
\end{array}$ & $\begin{array}{l}\text { Modelos de } \\
\text { negócio } \\
\text { sustentáveis }\end{array}$ & $\begin{array}{l}\text { Propõe exemplos de requisitos normativos que } \\
\text { os modelos de negócios devem atender para } \\
\text { apoiar inovações sustentáveis }\end{array}$ & Detalhado & - & 4 \\
\hline $\begin{array}{l}\text { Bocken et al. } \\
\quad \text { (2014) }\end{array}$ & $\begin{array}{l}\text { Modelos de } \\
\text { negócio } \\
\text { sustentáveis }\end{array}$ & $\begin{array}{l}\text { Incorpora o TBL, considerando as partes } \\
\text { interessadas, incluindo meio ambiente e } \\
\text { sociedade, considerando mecanismos } \\
\text { relacionados ao valor }\end{array}$ & Geral & 8 & 10 \\
\hline $\begin{array}{l}\text { Kindström e } \\
\text { Kowalkowski } \\
\quad(2014)\end{array}$ & $\begin{array}{l}\text { Inovação de } \\
\text { serviços }\end{array}$ & $\begin{array}{l}\text { Apresenta quais recursos e capacidades } \\
\text { exclusivos as empresas centradas em produto } \\
\text { devem desenvolver e implantar para buscar a } \\
\text { inovação de serviços }\end{array}$ & Detalhado & - & 10 \\
\hline $\begin{array}{l}\text { Gelbmann e } \\
\text { Hammerl } \\
(2015)\end{array}$ & $\begin{array}{l}\text { PSS para } \\
\text { reuso }\end{array}$ & $\begin{array}{l}\text { Apresenta uma estrutura para PSS para o reuso } \\
\text { (ECO-WISE) alinhado com o TBL destacando } \\
\text { a importância da integração com os } \\
\text { stakeholders }\end{array}$ & Detalhado & 6 & 6 \\
\hline $\begin{array}{l}\text { Reim et al. } \\
\quad(2015)\end{array}$ & PSS & $\begin{array}{l}\text { Descreve cada tipo de PSS (conforme } \\
\text { TUKKER, 2004) ligado a cinco táticas de nível } \\
\text { operacional e práticas relacionadas a } \\
\text { implementação de modelos de negócios PSS }\end{array}$ & Em tópicos & 3 & 5 \\
\hline $\begin{array}{l}\text { Zancul et al. } \\
\text { (2016) }\end{array}$ & IPSS & $\begin{array}{l}\text { Destaca o papel da tecnologia e Internet of } \\
\text { Things (IoT) nos modelos de negócio de PSS } \\
\end{array}$ & Descritivo & - & 7 \\
\hline $\begin{array}{l}\text { Adrodegari et } \\
\text { al. }(2017)\end{array}$ & IPSS & $\begin{array}{l}\text { Descreve a relevância de cada um dos } \\
\text { componentes do modelo Canvas para o PSS e } \\
\text { subdivide os componentes principais destacados } \\
\text { pelo modelo em tópicos }\end{array}$ & $\begin{array}{l}\text { Detalhado e } \\
\text { em tópicos }\end{array}$ & - & 8 \\
\hline $\begin{array}{l}\text { Fjeldstad e } \\
\text { Snow (2018) }\end{array}$ & $\begin{array}{l}\text { Modelo de } \\
\text { negócio e } \\
\text { estrutura da } \\
\text { organização }\end{array}$ & $\begin{array}{l}\text { Aponta que cada um dos elementos de um } \\
\text { modelo de negócios é afetado pela configuração } \\
\text { do valor da empresa, conectando o modelo de } \\
\text { negócios ao design da organização }\end{array}$ & Em tópicos & 3 & 8 \\
\hline
\end{tabular}

Fonte: quadro construído com base no portfólio e conteúdo das publicações

Apesar do foco principal ser em negócios relacionados com a internet, Osterwalder e Pigneur (2010) descrevem o processo de introdução no mercado da Car2Go (sistema de 
compartilhamento de carros - tradicional exemplo de PSS) como exemplo de inovação do modelo de negócio que complementa o modelo principal da Daimler. O modelo Canvas é frequentemente referenciado e considerado como um suporte padrão para o projeto de modelos de negócio, já que foi desenvolvido por meio de uma extensa investigação e representa o consenso de um grande grupo de especialistas da academia e da indústria (BARQUET et al., 2013). Nesse sentido, os componentes descritos nas publicações selecionadas foram comparados como os componentes do modelo Canvas de Osterwalder e Pigneur (2010), conforme apresenta o Quadro 3.

QUADRO 3 - Comparação entre os componentes dos modelos de negócio apresentados pela literatura.

\begin{tabular}{|c|c|c|c|c|c|c|c|c|c|c|}
\hline \multirow{2}{*}{ Publicação } & \multicolumn{9}{|c|}{ Componentes do modelo de negócio * } & \multirow{2}{*}{ Outros componentes } \\
\hline & $(1)$ & $(2)$ & (3) & (4) & $(5)$ & $(6)$ & (7) & $(8)$ & $(9)$ & \\
\hline $\begin{array}{l}\text { Meier et al. (2010); Meier } \\
\text { et al. (2011) }\end{array}$ & $\mathrm{x}$ & $\mathrm{x}$ & $\mathrm{x}$ & $\mathrm{x}$ & $\mathrm{x}$ & $\mathrm{x}$ & $\mathrm{x}$ & $\mathrm{x}$ & $\mathrm{x}$ & $\begin{array}{l}\text { - Valor para o consumidor } \\
\text { - Modelo de entrega do serviço } \\
\text { - Objetos/ recursos } \\
\text { - Processos }\end{array}$ \\
\hline Barquet et al. (2013) & $\checkmark$ & $\checkmark$ & $\checkmark$ & $\checkmark$ & $\checkmark$ & $\checkmark$ & $\checkmark$ & $\checkmark$ & $\checkmark$ & Não indicados pela publicação \\
\hline $\begin{array}{l}\text { Boons e Lüdeke-Freund } \\
(2013)\end{array}$ & $\mathrm{x}$ & $\checkmark$ & $\mathrm{x}$ & $\mathrm{x}$ & $\mathrm{x}$ & $\mathrm{x}$ & $\mathrm{x}$ & $\mathrm{x}$ & $\mathrm{x}$ & $\begin{array}{l}\text { - Interface com o cliente } \\
\text { - Cadeia de suprimentos } \\
\text { - Modelo financeiro }\end{array}$ \\
\hline Bocken et al. (2014) & $\checkmark$ & $\mathrm{x}$ & $\checkmark$ & $\checkmark$ & $\checkmark$ & $\checkmark$ & $\checkmark$ & $\checkmark$ & $\checkmark$ & $\begin{array}{l}\text { - Tecnologia } \\
\text { - Produto/serviço }\end{array}$ \\
\hline $\begin{array}{l}\text { Kindström e Kowalkowski } \\
(2014)\end{array}$ & $\mathrm{x}$ & $\mathrm{x}$ & $\mathrm{x}$ & $\checkmark$ & $\checkmark$ & $\mathrm{x}$ & $\mathrm{x}$ & $\mathrm{x}$ & $\mathrm{x}$ & $\begin{array}{l}\text { - Estratégia } \\
\text { - Estrutura } \\
\text { - Cultura } \\
\text { - Oferta } \\
\text { - Processo de vendas } \\
\text { - Processo de entrega } \\
\text { - Processo de desenvolvimento } \\
\text { - Rede de valor }\end{array}$ \\
\hline $\begin{array}{l}\text { Gelbmann e Hammerl } \\
(2015)\end{array}$ & $\mathrm{x}$ & $\checkmark$ & $\mathrm{x}$ & $\mathrm{x}$ & $\mathrm{x}$ & $\mathrm{x}$ & $\checkmark$ & $\mathrm{x}$ & $\mathrm{x}$ & $\begin{array}{l}\text { - Stakeholders } \\
\text { - Características-chave } \\
\text { - Relacionamento e parcerias } \\
\text { - Implicações financeiras }\end{array}$ \\
\hline Reim et al. (2015) & $\mathrm{x}$ & $\mathrm{x}$ & $\mathrm{x}$ & $\mathrm{x}$ & $\mathrm{x}$ & $\mathrm{x}$ & $\mathrm{x}$ & $\mathrm{x}$ & $\mathrm{x}$ & $\begin{array}{l}\text { - Sustentabilidade } \\
\text { - Marketing } \\
\text { - Contratos } \\
\text { - Projeto do produto e serviço } \\
\text { - Redes }\end{array}$ \\
\hline Zancul et al. (2016) & $\checkmark$ & $\checkmark$ & $\mathrm{x}$ & $\checkmark$ & $\checkmark$ & $\checkmark$ & $\mathrm{x}$ & $\mathrm{x}$ & $\checkmark$ & - Parcerias e atividades-chaves \\
\hline Adrodegari et al. (2017) & $\checkmark$ & $\checkmark$ & $\checkmark$ & $\mathrm{x}$ & $\checkmark$ & $\checkmark$ & $\checkmark$ & $\checkmark$ & $\checkmark$ & Não indicados pela publicação \\
\hline Fjeldstad e Snow (2018) & $\checkmark$ & $\checkmark$ & $\mathrm{x}$ & $\mathrm{x}$ & $\checkmark$ & $\checkmark$ & $\checkmark$ & $\mathrm{x}$ & $\mathrm{x}$ & $\begin{array}{l}\text { - Ofertas produto/serviço } \\
\text { - Economia } \\
\text { - Mecanismos de proteção }\end{array}$ \\
\hline
\end{tabular}

Fonte: quadro construído com base no portfólio e conteúdo das publicações

Nota: * Os números referem-se aos componentes apresentados na Figura 1, sendo: (1) - Segmentos de clientes;

(2) - Proposta de valor; (3) - Canais; (4) - Relacionamento com clientes; (5) - Fontes de receita; (6) Recursosprincipais; (7) - Atividades-chave; (8) - Parcerias principais; e (9) - Estrutura de custos. 
Com base na análise dos componentes, pode-se perceber que os componentes apresentados por Osterwalder e Pigneur (2010) estão presentes em grande parte dos modelos descritos por outros autores. Diversas publicações subsequentes aplicam o modelo Canvas de Osterwalder e Pigneur (2010) para o desenvolvimento de soluções PSS (e.g., BARQUET et al., 2013; FRANÇA et al., 2017), uma parte dos componentes (e.g., ZANCUL et al., 2016; ADRODEGARI et al., 2017) ou adicionam novos componentes (e.g., REIM et al., 2015; FJELDSTAD; SNOW, 2018). Outros componentes destacados por outros autores podem estar presentes em mais de um componente como, por exemplo, tecnologia (BOCKEN et al., 2014), sustentabilidade (REIM et al., 2015), e economia (FJELDSTAD; SNOW, 2018).

A literatura a respeito de modelos de negócio evidencia que o PSS é uma forma sustentável de oferecer valor para os clientes (BOONS; LÜDEKE-FREUND, 2013; BOCKEN et al., 2014). O PSS em sua origem tinha foco nas questões de sustentabilidade. Após a evolução do conceito, tornaram-se questões centrais a rentabilidade do modelo de negócio e a competitividade das empresas. Portanto, o PSS deve aliar as três dimensões da sustentabilidade: econômica, ambiental e social em uma abordagem holística, atendendo aos interesses das partes envolvidas (VASANTHA et al., 2012) e possuir foco na geração de valor.

\subsection{Mecanismos para a geração de valor do modelo de negócio de PSS}

Sem considerar o conceito de modelo de negócio, Pawar et al. (2009) identificaram etapas para criação de valor nas ofertas produto-serviço, sendo elas:

(i) definir o valor - identificar o valor do cliente, as necessidades e o custo de atendê-las para determinar a lucratividade de um PSS;

(ii) projetar o valor - identificar os requisitos organizacionais em termos de recursos que estão disponíveis ou são necessários; e

(iii) entregar o valor - selecionar a rede de parceiros que podem fornecer os recursos necessários e gerenciar o desempenho dessa rede para garantir acesso ininterrupto ao valor para os clientes. Evans et al. (2017) apontam que a internalização de externalidades por meio do PSS (e.g., manutenção, reparo, etc.) permite inovações para os modelos de negócio sustentáveis. As externalidades dos modelos de negócio baseados em produtos são trazidas para o núcleo do conceito de PSS. Os autores anteriormente citados apontam que para internalizá-las, é 
importante incorporar os conceitos de pensamento de ciclo de vida e estabelecer novos modos de propriedade que levem as empresas a maiores responsabilidades.

É consenso entre os principais autores, que a proposta de valor é o principal componente dos modelos de negócio (ADRODEGARI et al., 2017). No entanto, a proposta de valor é uma dificuldade ao desenvolver um modelo de negócio, que é abordada por Osterwalder et al. (2015), com foco específico em como desenvolver a proposição de valor de um modelo de negócio. Nesse sentido, Osterwalder et al. (2015) também colocam que, para auxiliar no desenvolvimento da proposta de valor do PSS, é preciso compreender as tarefas dos clientes. Ademais, é necessário que a proposição de valor comunique mudanças potencialmente favoráveis no valor para o cliente como problemas/sacrifícios reduzidos e benefícios (OSTERWALDER et al., 2015). Para desenvolver a proposta de valor podem ser utilizadas estratégias que integrem as considerações econômicas, ambientais e sociais, com foco na troca de valor. É importante que fique claro o benefício/valor que o cliente recebe (ADRODEGARI et al., 2017).

A criação de valor do modelo de negócio de PSS é responsável pela definição das principais características e qualidade do PSS (ANNARELLI et al., 2016). As melhorias no modelo de negócio não se limitam as áreas de produção da empresa, mas também em todas as atividades nas cadeias de valor do produto e fornecimento e podem emergir em qualquer ponto do ciclo de vida do PSS (VAN HALEN et al., 2005).

Os mecanismos para entrega de valor do PSS compreendem a descrição dos canais de distribuição, relacionamento com clientes e a eficiência dos processos. É necessário identificar as partes interessadas nos relacionamentos comerciais e desenvolver colaboração estreita entre o cliente e o fornecedor por meio de procedimentos interativos (VASANTHA et al., 2012).

Um modelo de negócio de PSS permite a coleta de dados a respeito do produto/serviço em uso e monitoramento de produtos na fase de uso (ZANCUL et al., 2016), facilitando o fornecimento de informações para modificações, redesenho de produtos e informações sobre o escopo do desenvolvimento de serviços necessários para atender às necessidades futuras dos clientes (PARIDA et al., 2014). Em relação à prática de PSS, a literatura mostra que os provedores de PSS têm lutado para capturar completamente os benefícios das ofertas de PSS (YANG et al., 2017). Outro fator importante a considerar é que os modelos de negócio sustentáveis requerem 
uma rede de valor com um novo propósito, projeto e governança, considerando os interesses e responsabilidades dos stakeholders na criação de valor mútuo (EVANS et al., 2017).

O Quadro 4 organiza os 14 componentes identificados como importantes para os modelos de negócio de PSS, em quatro etapas: (i) proposição, (ii) criação, (iii) entrega, e (iv) captura de valor.

QUADRO 4 - Contribuições dos modelos de negócio apresentados pela literatura.

\begin{tabular}{|c|c|c|}
\hline & $\begin{array}{l}\text { Componente do modelo de } \\
\text { negócio }\end{array}$ & Descrição \\
\hline \multirow{5}{*}{ 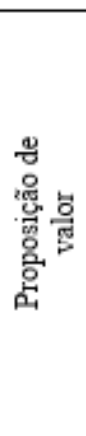 } & 1. Segmento de clientes & $\begin{array}{l}\text { Atendimento das necessidades de clientes segmentados de maneira } \\
\text { sustentável }\end{array}$ \\
\hline & 2. Tarefas do cliente & Funções e/ou situações para as quais o consumidor usará a solução PSS \\
\hline & 3. Dores/ problemas do cliente & $\begin{array}{l}\text { Incorpora a solução de problemas dos clientes nas três dimensões da } \\
\text { sustentabilidade }\end{array}$ \\
\hline & $\begin{array}{l}\text { 4. Ganhos/ beneficios para o } \\
\text { cliente }\end{array}$ & $\begin{array}{l}\text { Representa o beneficio pelo qual o cliente está disposto a pagar } \\
\text { Oferece valor agregado por meio da ênfase em menor preço e melhor } \\
\text { qualidade }\end{array}$ \\
\hline & 5. Proposta de valor & $\begin{array}{l}\text { Descreve as suposições de seus propósitos para os consumidores, } \\
\text { diferenciando-os dos concorrentes e sustentando suas atividades e uso de } \\
\text { recursos } \\
\text { Representada pela troca de valor }\end{array}$ \\
\hline \multirow{3}{*}{ 惢 } & 6. Recursos principais & Maximização do uso dos recursos durante todo o ciclo de vida do PSS \\
\hline & 7. Atividades principais & Foco na longevidade dos produtos e eco eficiência do sistema \\
\hline & 8. Parcerias principais & $\begin{array}{l}\text { Atividades e recursos que vão além dos limites da empresa, incluindo } \\
\text { parcerias entre partes interessadas, tais como produtores, revendedores, } \\
\text { clientes e "administradores de fim de vida dos produtos' com interesses } \\
\text { econômicos conectados }\end{array}$ \\
\hline \multirow{3}{*}{ 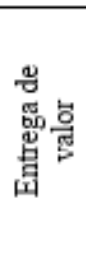 } & 9. Canais de distribuição & Busca enfatizar o valor entregue, melhorando a eficiência do sistema \\
\hline & 10.Relacionamento com clientes & $\begin{array}{l}\text { Estabelecimento de contato direto com o consumidor } \\
\text { Eficácia do suporte e resolução de problemas }\end{array}$ \\
\hline & 11.Indicadores de eficiência & $\begin{array}{l}\text { Receptividade e aceitação dos clientes } \\
\text { Indices de desperdicios, emissões e poluição } \\
\text { Tempo de resposta e atendimento ao cliente } \\
\text { Durabilidade dos produtos e eficiência da manutenção }\end{array}$ \\
\hline \multirow{3}{*}{ 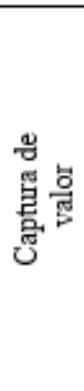 } & 12.Fontes de receita & $\begin{array}{l}\text { Os consumidores pagam pelo uso do serviço e/ou pelo trabalho feito } \\
\text { Extensão de ofertas existentes } \\
\text { Foco na extensão do prazo de contração do PSS }\end{array}$ \\
\hline & 13.Estrutura de custos & $\begin{array}{l}\text { O custo do PSS deve ser comparado ao valor fornecido } \\
\text { O custo da posse dos produtos fisicos é suportado pela empresa e seus } \\
\text { parceiros } \\
\text { Responsabilidade pelas questões econômicas, ambientais, e sociais durante e } \\
\text { após a fase de uso do produto } \\
\text { Foco na redução do uso de recursos e eficácia do sistema }\end{array}$ \\
\hline & 14.Rede de valor & $\begin{array}{l}\text { Rede integrada de fomecedores com papéis definidos claramente } \\
\text { Rentabilidade para todos os envolvidos }\end{array}$ \\
\hline
\end{tabular}

Fonte: quadro elaborado pelos autores com base na análise da literatura

Apesar de seguir uma sequência lógica, o processo de estruturação do modelo de negócio PSS deve ser cíclico e iterativo e não sequencial e linear (TUKKER, 2015). Por fim, salienta-se que a economia global é cada vez mais digital, em rede e baseada no conhecimento, o que exige que as empresas reconsiderem constantemente seus modelos de negócio e, se necessário, os 
modifiquem, para se adaptarem às condições de mudança (FJELDSTAD; SNOW, 2018). Sendo assim, é necessário que ocorra a integração e troca de informações entre os stakeholders, de modo que a sustentabilidade do modelo de negócio inclua todos os envolvidos na rede de valor.

\section{SÍNTESE CONCLUSIVA}

O conceito de modelo de negócio é ainda um pouco recente e nas pesquisas em PSS, o interesse aumentou nos últimos anos. Percebe-se que a estruturação do PSS utilizando o conceito de modelo de negócio pode favorecer o seu entendimento e facilitar as atividades de desenvolvimento e posterior implementação. Com base na análise realizada foram apontados 14 componentes para serem considerados ao estruturar um modelo de negócio, organizados em mecanismos de proposição, criação, entrega, e captura de valor. Uma visão centrada no valor é essencial para que o modelo de negócio atenda às necessidades dos clientes, e aos interesses dos integrantes da rede de valor do PSS de maneira sustentável.

Como limitação do presente trabalho cita-se que a análise cobriu apenas a visão dos pesquisadores. Ao considerar o PSS como um modelo de negócio, é importante também considerar a literatura sobre servitização, uma vez que esta se refere ao processo de criação de fluxos de receita para organizações de serviços. Assim, uma oportunidade de pesquisa futura seria incluir palavras relacionadas a servitização, visando ampliar o escopo. O trabalho não cobriu também a análise dos dados demográficos das publicações, que pode também revelar dados relevantes acerca da evolução da literatura.

\section{AGRADECIMENTOS}

Os autores agradecem ao CNPq pelo apoio financeiro - processos 428946/2018-6 (Chamada Universal) e 303538/2016-3 (produtividade em pesquisa), e bolsa de doutorado.

\section{REFERÊNCIAS}

ADRODEGARI, F.; SACCANI, N.; KOWALKOWSKI, C.; VILO, J. PSS business model conceptualization and application. Production Planning \& Control, v. 28, n. 15, p. 1251-1263, 2017.

ANNARELLI, A.; BATTISTELLA, C.; NONINO, F. Product service system: A conceptual framework from a systematic review. Journal of Cleaner Production, v. 139, p. 1011-1032, 2016.

BARQUET, A.P.B.; DE OLIVEIRA, M.G.; AMIGO, C.R.; CUNHA, V.P.; ROZENFELD, H. Employing the business model concept to support the adoption of product-service systems (PSS). Industrial Marketing Management, v. 42, n. 5, p. 693-704, 2013.

BOCKEN, N.M.P; SHORT, S.W.; RANA, P.; EVANS, S. A literature and practice review to develop sustainable business model archetypes. Journal of Cleaner Production, v. 65, p. 42-56, 2014. 
BOONS, F.; LÜDEKE-FREUND, F. Business models for sustainable innovation: state-of-the-art and steps towards a research agenda. Journal of Cleaner Production, v. 45, p. 9-19, 2013.

BOOTH, A.; SUTTON, A.; PAPAIOANNOU, D. Systematic approaches to a successful literature review. Sage, 2012.

CHESBROUGH, H.; ROSENBLOOM, R.S. The role of the business model in capturing value from innovation: evidence from Xerox Corporation's technology spin-off companies. Industrial and Corporate Change, v. 11, n. 3, p. 529-555, 2002.

EVANS, S.; VLADIMIROVA, D.; HOLGADO, M.; VAN FOSSEN, K.; YANG, M.; SILVA, E.A.; BARLOW, C.Y. Business model innovation for sustainability: Towards a unified perspective for creation of sustainable business models. Business Strategy and the Environment, v. 26, n. 5, p. 597-608, 2017.

FJELDSTAD, Ø. D.; SNOW, C.C. Business models and organization design. Long Range Planning, v. 51, n. 1, p. 32-39, 2018.

FRANÇA, C.L.; BROMAN, G.; ROBÈRT, K.H.; BASILE, G.; TRYGG, L. An approach to business model innovation and design for strategic sustainable development. Journal of Cleaner Production, v. 140, p. 155-166, 2017.

GELBMANN, U.; HAMMERL, B. Integrative re-use systems as innovative business models for devising sustainable product-service-systems. Journal of Cleaner Production, v. 97, p. 50-60, 2015.

GOUGH, D.; OLIVER, S.; THOMAS, J. (Ed.). An introduction to systematic reviews. SAGE Publications Ltd, 2012.

KINDSTRÖM, D.; KOWALKOWSKI, C. Service innovation in product-centric firms: A multidimensional business model perspective. Journal of Business \& Industrial Marketing, v. 29, n. 2, p. 96-111, 2014.

MAHUT, F.; DAABOUL, J.; BRICOGNE, M.; EYNARD, B. Product-Service Systems for servitization of the automotive industry: a literature review. International Journal of Production Research, v. 55, n. 7, p. 2102$2120,2017$.

MEIER, H.; ROY, R.; SELIGER, G. Industrial product-service systems - IPS2. CIRP Annals-Manufacturing Technology, v. 59, n. 2, p. 607-627, 2010.

MEIER, H.; VÖLKER, O.; FUNKE, B. Industrial product-service systems (IPS 2). The International Journal of Advanced Manufacturing Technology, v. 52, n. 9-12, p. 1175-1191, 2011.

OSTERWALDER, A. The business model ontology a proposition in a design science approach. 2004. Tese de Doutorado. Université de Lausanne, Faculté des hautes études commerciales.

OSTERWALDER, A.; PIGNEUR, Y. Business Model Generation: a handbook for visionaries, game changers, and challengers. John Wiley \& Sons, 2010.

OSterWalder, A.; PIGNEUR, Y.; BERnARDA, G.; SMITH, A. Value Proposition Design. Campus Verlag, 2015.

OSTERWALDER, A.; PIGNEUR, Y; TUCCI, C.L. Clarifying business models: Origins, present, and future of the concept. Communications of the Association for Information Systems, v. 16, n. 1, p. 1, 2005.

PARIDA, V.; SJÖDIN, D.R.; WINCENT, J.; KOHTAMÄKI, M. Mastering the transition to product-service provision: Insights into business models, learning activities, and capabilities. Research-Technology Management, v. 57, n. 3, p. 44-52, 2014.

PAWAR, K.S.; BELTAGUI, A.; RIEDEL, J.C.K.H. The PSO triangle: designing product, service and organisation to create value. International Journal of Operations \& Production Management, v. 29, n. 5, p. 468-493, 2009.

REIM, W.; PARIDA, V.; ÖRTQVIST, D. Product-Service Systems (PSS) business models and tactics-a systematic literature review. Journal of Cleaner Production, v. 97, p. 61-75, 2015.

SCHEEPENS, A.E.; VOGTLÄNDER, J.G.; BREZET, J.C. Two life cycle assessment (LCA) based methods to analyse and design complex (regional) circular economy systems. Case: making water tourism more sustainable. Journal of Cleaner Production, v. 114, p. 257-268, 2016. 
SHORT, S.W.; BOCKEN, N.M.; BARLOW, C.Y.; CHERTOW, M.R. From refining sugar to growing tomatoes. Journal of Industrial Ecology, v. 18, n. 5, p. 603-618, 2014.

TEECE, D.J. Business models, business strategy and innovation. Long Range Planning, v. 43, n. 2-3, p. $172-$ 194, 2010.

TUKKER, A. Eight types of product-service system: eight ways to sustainability? Experiences from SusProNet. Business Strategy and the Environment, v. 13, n. 4, p. 246-260, 2004.

TUKKER, A. Product services for a resource-efficient and circular economy-a review. Journal of Cleaner Production, v.97, p.76-91, 2015.

VAN HALEN, C.; VEZZOLI, C.; WIMMER, R. Methodology for product service system innovation: how to develop clean, clever and competitive strategies in companies. Uitgeverij Van Gorcum, 2005.

VASANTHA, G.V.A.; ROY, R.; LELAH, A.; BRISSAUD, D. A review of product-service systems design methodologies. Journal of Engineering Design, v. 23, n. 9, p. 635-659, 2012.

YANG, M.; EVANS, S.; VLADIMIROVA, D.; RANA, P. Value uncaptured perspective for sustainable business model innovation. Journal of Cleaner Production, v. 140, parte 3, p. 1794-1804, 2017.

ZANCUL, E.D.S.; TAKEY, S.M.; BARQUET, A.P.B.; KUWABARA, L.H.; CAUCHICK MIGUEL, P.A.; ROZENFELD, H. Business process support for IoT based product-service systems (PSS). Business Process Management Journal, v. 22, n. 2, p. 305-323, 2016. 